\title{
2-D shape completion with shape priors
}

\author{
GUO Ge $\mathrm{G}^{1,2}$, JIANG TingTing ${ }^{3 *}$, WANG YiZhou ${ }^{3} \&$ GAO Wen ${ }^{3}$ \\ ${ }^{1}$ Key Laboratory of Intelligent Information Processing, Institute of Computing Technology, Chinese Academy of Sciences, Beijing 100190, China; \\ ${ }^{2}$ Graduate University of Chinese Academy of Sciences, Beijing 100049, China; \\ ${ }^{3}$ National Engineering Laboratory for Video Technology, Key Laboratory of Machine Perception, School of EECS, Peking University, \\ Beijing 100871, China
}

Received November 21, 2012; accepted April 1, 2013

\begin{abstract}
We propose new techniques for 2-D shape/contour completion, which is one of the important research topics related to shape analysis and computer vision, e.g. the detection of incomplete objects due to occlusion and noises. The purpose of shape completion is to find the optimal curve segments that fill the missing contour parts, so as to acquire the best estimation of the original complete object shapes. Unlike the previous work using local smoothness or minimum curvature priors, we solve the problem under a Bayesian formulation taking advantage of global shape prior knowledge. With the priors, our methods are expert in recovering significant shape structures and dealing with large occlusion cases. There are two different priors adopted in this paper: (i) A generic prior model that prefers minimal global shape transformation (including non-rigid deformation and affine transformation with respect to a reference object shape) of the recovered complete shape; and (ii) a class-specific shape prior model learned from training examples of an object category, which prefers the reconstructed shape to follow the learned shape variation models of the category. Efficient contour completion algorithms are suggested corresponding to the two types of priors. Our experimental results demonstrate the advantage of the proposed shape completion approaches compared to the existing techniques, especially for objects with complex structure under severe occlusion.
\end{abstract}

curve completion, contour completion, shape completion, shape reconstruction, Bayesian model

Citation: Guo G, Jiang T T, Wang Y Z, et al. 2-D shape completion with shape priors. Chin Sci Bull, 2013, 58: 3430-3436, doi: 10.1007/s11434-013-5946-4

Shape is an important cue for object recognition and popularly used in many computer vision tasks. There is a large body of literature about shape-related approaches. However, incomplete object contours or shapes are frequently encountered due to occlusion, degradation and clutters in natural images. This will lead to the changes of object shapes, bring about great challenges and decrease the performance of shape matching, object detection and localization, etc.

Nevertheless, human vision is quite accustomed to incomplete shapes. There are scientific evidences about the powerful ability of our visual system to perform shape completion; early vision can rapidly recover the whole shape when it is partially occluded [1]. Therefore, shape completion is an indispensable and important research topic for shape analysis.

*Corresponding author (email: ttjiang @ pku.edu.cn)
What's more, many applications benefit from shape completion techniques. It serves for the tasks of filling in the occluded image regions. It can help to improve image inpainting and figure-ground segmentation. The researches on contour grouping from cluttered edge maps also take advantage of curve completion. Besides it is applied in 3-D reconstruction, mechanical design, engineering projects etc.

In the literature of planer shape completion, different kinds of curves have been utilized to optimize the bridging of two broken ends [2-4]. Some simple generic constraints have been proposed such as isotropy, smoothness, extensibility and locality. One important and widely used criterion is curvature-based constraint, such as the total curvature adopted by the elastica model and its variants [5-8], and variation in curvature used by the Euler Spirals [9]. There is also biologically-inspired curve completion [10] using the minimum length in the tangent bundle as a model. However 
these approaches are suitable for simple and smooth contours. For shapes of complex structures, they are not informative enough (Figure 1(a),(d)). In addition, it is proposed in [11] that symmetry of shapes can also be utilized for contour completion, but this is not universal for all shapes.

The amodal completion [12,13] is another group which specializes in the subjective contours, "illusory contours", for the cases that the occluders are blended with the background. In these studies, objects are usually very simple shapes such as triangles and disks, and the research is more concerned about generic visual perception cues for grouping-e.g. continuation, proximity, symmetry-rather than curve completion. Also, there are researches in finding salient contour boundaries in cluttered edge maps. The basic approach is to group a set of boundary fragments based on the statistics of continuity and different junction types [14], or taking advantage of convexity prior [15]. However it is difficult for them to handle complex shape classes with large occlusion.

Besides the local generic priors for shape completion, global shape priors are also proposed and incorporated in applications such as object segmentation under occlusion, e.g. the shape level sets [16]. Although the purpose of the work is not shape completion, object boundaries are recovered by balancing the global shape prior and image evidence terms in level set functions. However, it is known that the performance of the level-sets-based methods always relies on good initializations, and the methods are easy to be stuck in local optimum. While in our methods global shape deformation is estimated based on local/observed part deformation. We find analytical solutions in a global optimization framework.

In addition to planer shapes, there is related work on 3-D curve completion [17] and surface reconstruction [18]. Some use generic priors [19,20], such as smoothness and the regularity of the distribution of 3-D points. However, using only such global priors always results in losing shape details unique to objects. So it is proposed to consider the locality for shape reconstruction, making use of context information [18], local shape priors [21] and primitive shapes [22], etc. Some others adopt class-specified methods [23], in which the 3-D models of objects (e.g. human body) are learned in advance. Their primary goal is to reconstruct surfaces among the sparse 3-D points, whereas the goal of the proposed methods is to recover significant missing parts from observed fragments. The work in [23] inspires one of the proposed methods but ours uses a less constraint prior.

In this paper, we focus on shape completion of 2-D object contours by utilizing stronger global shape prior knowledge. The problem is formulated under a Bayesian framework. Given an incomplete shape as observation, our goal is to infer the optimal complete shape, which should fit the observed part as well as possible, and meanwhile satisfy the prior constraints. Under this framework, two types of priors are introduced: (i) A generic prior model and (ii) a class-specific prior model. Firstly, in the generic prior model, it constrains that the recovered complete shape should have minimal global shape transformation-including the non-rigid shape deformation and affine transformation with respect to a reference object shape. Secondly, the classspecific prior model is learned from a database where each object class contains a set of training examples. It prefers the reconstructed shape to follow the learned shape variation models of the object class. We propose efficient contour completion algorithms corresponding to the two types of priors. The experimental results demonstrate that both approaches reconstruct object shapes more accurately than using the existing methods, especially with large part of occlusion.

\section{The Bayesian model for shape completion}

In the problem of shape completion, the observed data are the incomplete object contours, usually some curve segments. Based on the observation, our purpose is to estimate the complete object contour in a perceptually consistent manner. It is very difficult to solve this under-constraint problem, especially in the case of severe occlusion.

This paper focuses on the completion of 2-D object shapes, which is formulated under the Bayesian framework. Suppose that the observation i.e. the incomplete shape $x$ comes from a certain complete shape $y$. In statistical models $x$ is supposed to stem from the underlying shape $y$ following the probability model $x \sim p(x \mid y)$, where $p(x \mid y)$ is the probability that $x$ comes from $y$. The goal is to infer the unknown variable $y$, which is formulated as a Maximum a

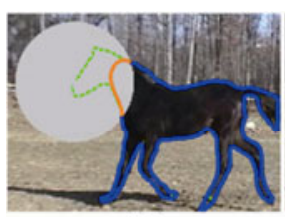

(a)

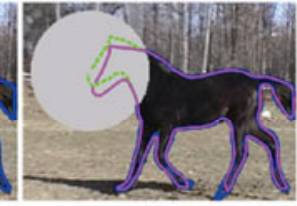

(b)

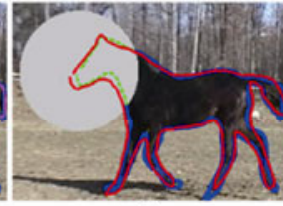

(c)

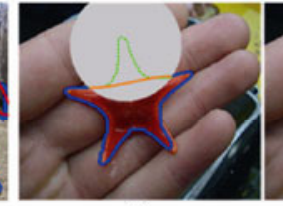

(d)

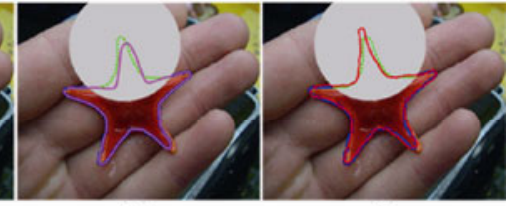

(e)

(f)

Figure 1 Illustration of shape completion. The blue curves are the observed object contours. The green dot curves are the occluded ground-truth boundaries. The orange, pink and red curves are the results of shape completion based on the Euler spirals in [9] ((a) and (d)), and our proposed generic ((b) and (e)) and class-specific approaches ((c) and (f)) respectively. 
Posteriori (MAP) problem,

$$
y^{*}=\arg \max _{y \in \Omega_{R^{2}}} p(y \mid x)=\arg \max _{y \in \Omega_{R^{2}}} \frac{p(x \mid y) p(y)}{\int p(x \mid y) p(y) \mathrm{d} y},
$$

where $\Omega_{R^{2}}$ denotes the space of 2-D shapes.

The underlying complete shape $y$ to be inferred should satisfy some constraints. For example, many previous scientists draw the assumption that it should belong to the group of smooth curves. This is actually adding a window function on the prior of $y$, in which only smooth curves have nonzero priors. However in real applications, we are confronting much more complicated shapes than the smooth curves, especially for those contours with significant shape structures and features (such as sharp corners and long protuberances). In such cases, using the smoothness prior would have obvious limitations.

Our idea in this paper is to reconstruct complete object contour by taking advantage of our knowledge on common object shapes. This is inspired by human visual experiences. For example, if a partially occluded object contour is observed, we can imagine what the rest part of the shape would be like with our knowledge of the object shape. It is unlikely for us to reconstruct a visually inexperienced shape. Therefore our shape completion can be implemented based on a database $\Omega_{S}$, which consists of categories of object shapes that we have known, $\Omega_{S} \subset \Omega_{R^{2}}$. The reconstructed shapes are variants of these common shapes $s$ in the dictionary $\Omega_{S}=\left\{s_{i}\right\}$. Then the posterior in eq. (1) is computed by

$$
p(y \mid x)=\sum_{i} p\left(y \mid x, s_{i}\right) p\left(s_{i} \mid x\right)
$$

This is actually considered as a Gaussian mixture model, where $p\left(s_{i} \mid x\right)$ is the probability of selecting shape $s_{i}$ to complete the occluded shape $x$; and it is in fact the weight of the Gaussian models.

We consider two scenarios for this problem. The first is a Generic Shape Database (called GSD for short), which is a set of typical shapes of different object classes (Figure 2(a)). And the second is a Class-specific Shape Database (called CSD) with rich shape information of object categories, in which each object class has various shape instances (Figure 2(b)). Based on the CSD, we can learn the class-specific shape models. Currently both datasets includes categories as that of ETHZ datasets [24] and Weizmann horses [25], which are prevalent shape benchmarks. Although ideally it seems that we should exhaust as many object categories as possible to compute eq. (2), it is noticed that quite a lot of classes are irrelevant, since the observed part is of little possibility to come from a class with far different shapes. So in fact, in most cases our goal is to approximate the problem as follows,

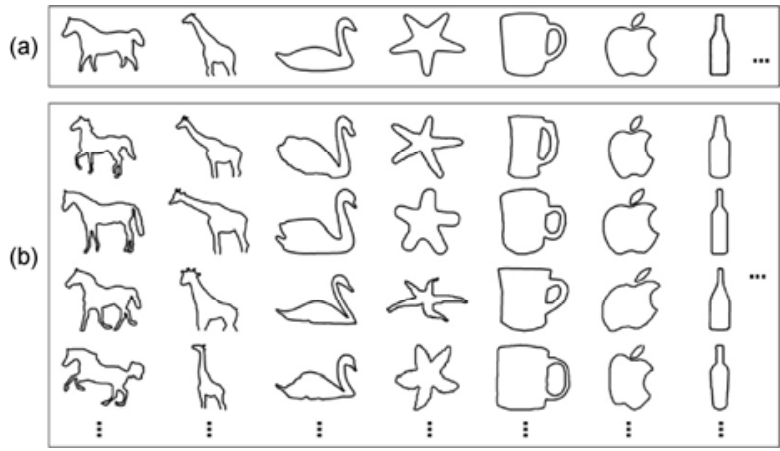

Figure 2 Examples of the databases (a) GSD and (b) CSD.

$$
p(y \mid x)=\sum_{i} p\left(y \mid x, s_{i}\right) p\left(s_{i} \mid x\right) \approx p\left(y \mid x, s_{i}^{*}\right) p\left(s_{i}^{*} \mid x\right),
$$

where the shape class $s_{i}^{*}$ is the one leading to the highest reconstruction probability. We can primarily select those classes with similar shape parts to the observed data, and finally find the optimal reference class and reconstruction.

For a possible reference class used to reconstruct the shape $y$ given $x$, there is a hidden variable indicating the correspondence between the part and the whole,

$$
\begin{aligned}
p\left(y \mid x, s_{i}\right) & =\sum_{l} p\left(y \mid x, s_{i}, l\right) p\left(l \mid s_{i}\right) \\
& \approx \sum_{l} p\left(x \mid y, s_{i}, l\right) p\left(y \mid s_{i}\right) p\left(l \mid s_{i}\right),
\end{aligned}
$$

where $p\left(x \mid y, s_{i}, l\right)$ describes the partial consistency between $x$ and the part at location $l$ of the whole shape, and $p\left(y \mid s_{i}\right)$ is the prior. In practice only those good partial matches are considered, since otherwise the likelihood $p\left(x \mid y, s_{i}, l\right)$ will be very low.

In the following we will introduce the two shape completion approaches based on the GSD and CSD in Sections 1.1 and 1.2 respectively, with the knowledge of a possible reference class and the possible partial correspondence.

\subsection{Shape completion based on GSD}

The Generic Shape Database is a world of familiar shapes of different object classes. For each object class $c$ there is just one example shape $s_{c}$ included, which is considered as a possible reference shape, as shown in Figure 2(a).

Based on the reference shape $s_{c}$, there are two modules to solve the problem (as illustrated in Figure 3), partial shape matching and global transformation estimation. At first, we compute the transformation (including the affine transformation and warping deformation) between the incomplete data and its matched part on the reference shape under a possible correspondence $l$. Secondly, we infer the global optimal transformation especially the shape warping deformation according to the partial transformation. A classical shape matching algorithm TPS-RPM [26] is utilized to infer 
the transformation between two shapes.

We adopt the point-based representation for shape contours throughout the paper. Let $X, Y$ and $S$ denote the point sets of the shape $x, y$ and $s_{c}$ respectively. For the partial shape matching, let $S_{p}$ be the part of the reference shape matching with $X$ under the correspondence $l$. The affine transformation $A_{P}$ and warping deformation $D_{P}$ between $S_{P}$ and $X$ are calculated by [26].

For the global transformation estimation, suppose $A$ and $D$ are the global affine transformation and deformation between the reference shape $S$ and the target complete shape $Y$ respectively. We have

$$
\begin{gathered}
Y=S \cdot A+K \cdot D, \\
\left(\begin{array}{l}
Y_{P} \\
Y_{R}
\end{array}\right)=\left(\begin{array}{c}
S_{P} \\
S_{R}
\end{array}\right) \cdot A+\left(\begin{array}{cc}
K_{P} & B \\
B^{\mathrm{T}} & K_{R}
\end{array}\right) \cdot\left(\begin{array}{c}
D_{P}^{\prime} \\
D_{R}
\end{array}\right),
\end{gathered}
$$

where $K$ is the TPS kernel generated from $S$. And $S_{R}=S \backslash S_{P}, K_{P}$ and $K_{R}$ are the TPS kernels from $S_{P}$, $S_{R}$ respectively. $D$ consists of $D_{P}^{\prime}$ and $D_{R}$, and we allow $D_{P}^{\prime}$ to be slightly different from the original $D_{P} . Y_{P}$ and $Y_{R}$ represent the completed shape parts corresponding to the observed shape and the unknown part respectively.

According to eq. (4),

$$
p\left(y \mid x, s_{c}, l\right) p\left(y \mid s_{c}\right)=p\left(X \mid Y_{P}, S_{P}\right) p(Y \mid S) \propto e^{-E_{P}-\lambda E_{G}},
$$

where $E_{P}=\left\|Y_{P}-X\right\|^{2}+\lambda_{1}\left\|D_{P}^{\prime}-D_{P}\right\|$,

$$
E_{G}=\left\|A-A_{P}\right\|^{2}+\lambda_{2} \text { trace }\left\|D^{\mathrm{T}} K D\right\| .
$$

$E_{P}$ accounts for the inconsistency of the completed shape with the observation. It is measured by the distance between the corresponding shape points, and the change of the partial wrapping; $E_{G}$ is the global shape transformation energy, which constraints that the global affine transformation between $Y$ and $S$ is consistent with the partial affine transformation $A_{P}$, and the amount of the total deformation should not be large. The parameters $\lambda_{1}$ and $\lambda_{2}$ balance the energy terms.

We solve the shape completion problem by minimizing the energy function $E=E_{P}+\lambda E_{G}$. Our goal is to estimate the optimal $A, D_{P}^{\prime}$ and $D_{R}$ and reconstruct the best completed shape $Y$ according to eq. (5). And the energy minimization problem is solved by the methods such as least square and gradient-descent.

\subsection{Shape completion based on CSD}

In the Class-specific Shape Database, each object class $c$ has a number of training examples $s_{c}^{j}, j \in\left\{1, \cdots, n_{c}\right\}$, where $n_{c}$ is the number of training data of class $c$, as shown in Figure 2(b). For each class the shape model $M_{c}$ can be learned based on the ASM approach [27]. In this case we take advantage of the learned shape model to solve the shape com- pletion problem.

Similarly to the GSD-based method in section 1.1, the posterior with respect to the class $c$ is

$$
p\left(y \mid x, s_{c}^{j}, l\right) p\left(y \mid s_{c}^{j}\right)=p\left(X \mid Y, M_{c}, l\right) p\left(Y \mid M_{c}\right) \propto \mathrm{e}^{-E_{P}^{\prime}-\lambda^{\prime} E_{G}^{\prime}} .
$$

There are still two constraints. One is the partial shape consistency which corresponds to the term $p\left(X \mid Y, M_{c}, l\right)$ and similar to that of the GSD; and the other is the prior that the reconstructed shape $Y$ is expected to follow the shape model of the class, corresponding to the term $p\left(Y \mid M_{c}\right)$.

According to the ASM, let $S$ be the mean shape in the point-based representation, and $\Phi$ be the matrix consisting of the eigenvectors learned from the training data. Any shape $\mathrm{Y}$ can be projected into the PCA space as

$$
\beta=\Phi^{\mathrm{T}}(Y-S) \text {. }
$$

The probability $p\left(Y \mid M_{c}\right)$ is considered to follow the Gaussian distribution in the PCA space, i.e. $\beta \sim N(0, \Sigma)$, where $\Sigma$ is the covariance matrix that can be learned from the training data. Accordingly we have

$$
E_{G}^{\prime}=\beta^{\mathrm{T}} \Sigma^{-1} \beta, \quad E_{P}^{\prime}=\left\|Y_{P}-X\right\|^{2} .
$$

During the ASM learning, all the training data are aligned, and we can obtain $\Phi_{P}$ which is learned from the aligned parts corresponding to the observation $X$. As a result $Y_{P}$ can be approximated by

$$
Y_{P}=S_{P}+\Phi_{P} \beta
$$

The goal is to find the optimal $\beta$ by minimizing $E^{\prime}=E_{P}^{\prime}+\lambda^{\prime} E_{G}^{\prime}$ using the least square method. Finally $Y$ is acquire by

$$
Y=S+\Phi \beta
$$

\section{Experiments and applications}

This section demonstrates the great reconstruction power, good completion results as well as the comparisons of the GSD- and CSD-based approaches. In addition, we apply the proposed reconstruction methods to occluded object detection, which show the advantages of shape completion to handle occlusion compared with the state-of-the-art method.

\subsection{Shape completion results}

Due to the lack of occlusion benchmark (to our best knowledge), we first experiment on the synthesized occlusion datasets, which are generated from the standard shape datasets by randomly placing ellipse boards of random size and aspect ratio into the test images, producing a variety of occlusion cases happening at different positions. One ad- 
vantage of the synthesized occlusion datasets is that, with the provided ground-truth outlines from the standard datasets, we can calculate the reconstruction accuracies for analytical evaluation of the reconstruction qualities. We use ETHZ datasets (the extended version) where approximately 40-80 images included in each class, and Weizmann-horses where 80 images of it are randomly selected. In the GSDbased approach, some hand-labeled shapes are employed for reference in our experiments (Figure 2(a)). In fact anyone of the object shapes from different classes can be utilized as the reference shape. In the CSD-based approach, the outlines of the object contours are taken as training examples to learn the shape model. The test experiments are implemented in a "leave-one-out" way to make use of as many training data as possible.

Figures 1 and 4 illustrate that by utilizing stronger shape prior knowledge, the proposed methods not only better reconstruct the complex shape structures, but also recover those nearly smooth contours more accurately than the traditional curvature-based approaches such as the Euler spirals [9]. Table 1 shows the statistics of shape completion errors, which is calculated by the root-mean-square error of the Euclidian distances between the corresponding points of the completed shapes and the ground-truth outlines. In the evaluation all the shapes are normalized to a comparable coordinate range, i.e. the longest side of the bounding box of each shape scaled into the range $[-1,1]$. It demonstrates that the proposed methods outperform the previous work using Euler spirals [9]. What's more, the GSD-based and CSD-based approaches achieve approximately the same level of completion errors, although the CSD-based method is more accurate and robust compared with the GSD. The parameters of the GSD approach in eqs. (6) and (7) are set to be $\lambda=\lambda_{1}=20, \lambda_{2}=0.005$. In the CSD-based

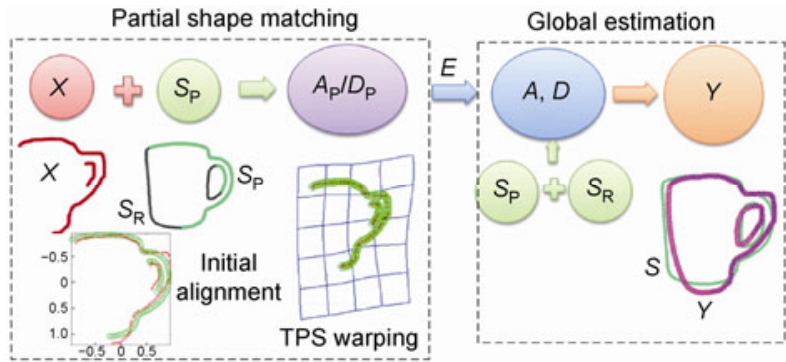

Figure 3 Illustration of the GSD-based shape completion. In the partial matching, the incomplete contour $X$ is matching with the part $S_{P}$ of the reference shape, and the transformation $\left(A_{P}, D_{P}\right)$ between the two is computed. In the global estimation, the optimized global transformation $(A, D)$ is estimated, and the complete shape $Y$ is reconstructed by transforming the reference shape $S=S_{P} \cup S_{R}$ according to $(A, D)$.

approach, the parameter $\lambda^{\prime}=0.01$.

From the experimental results, we observe that there are mainly two factors that affect the performances of shape completion. One is the amount of occlusion, and the other is shape variation of the observed contour. We illustrate how these two factors work on the completion quality, as shown in Figure 5. First, the amount of occlusion is $R=1-R_{0}$, where $R_{0}$ is the ratio of the curve length of the observed part to that of the entire ground-truth shape contour. We collect all the test data for all classes with different occlusion amount to draw the curve as in Figure 5(a). Generally the completion error increases as the occluded part becomes larger. Second, under the same amount of occlusion, we examine the relations between the completion errors and shape variations of the observed parts. The amount of shape variation is computed according to the ASM method, where the learning is with respect to the contour parts of the training data corresponding to the observed incomplete contour. It can be observed from Figure 5(b) and (c) that the more

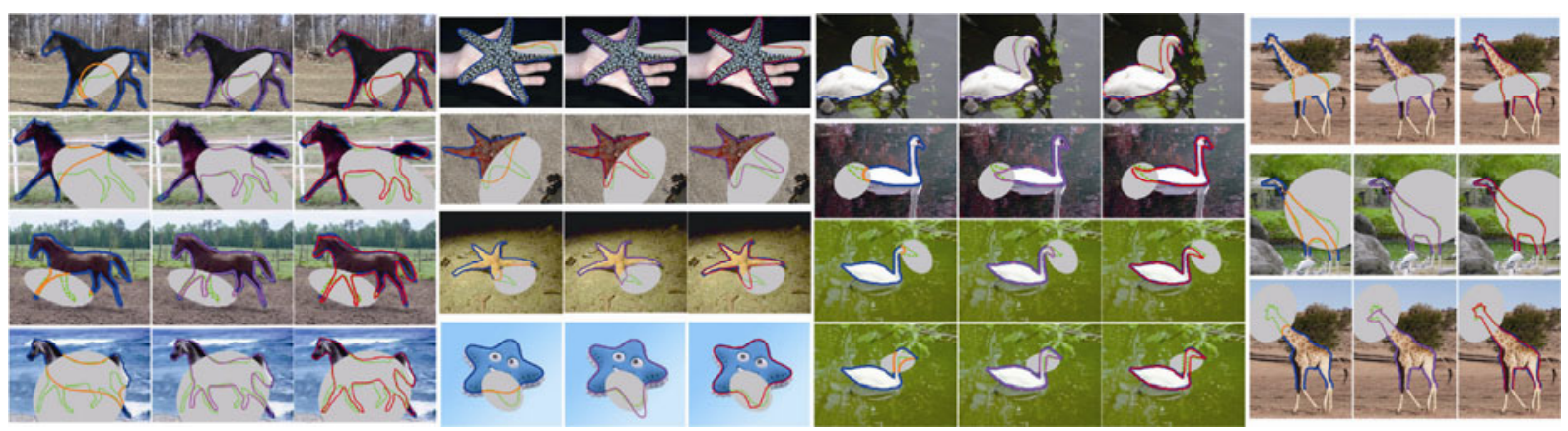

Figure 4 Shape completion results. The blue curves are the observed object contours. The green dot curves are the occluded ground-truth boundaries. The orange, pink and red curves are the results of shape completion based on Euler spirals in [9] and our proposed GSD- and CSD-based approaches respectively.

Table 1 The comparisons of completion errors (the mean value of each class) by the Euler spiral [9] and our proposed GSD- and CSD-based methods

\begin{tabular}{lccccccc}
\hline & Horses & Swans & Giraffes & Mugs & Bottles & Applelogos & Starfishes \\
\hline Euler spiral [9] & 0.7021 & 0.9206 & 0.9689 & 0.6771 & 0.3392 & 0.4826 & 0.7319 \\
GSD-based & 0.1949 & 0.2041 & 0.1960 & 0.1053 & 0.0474 & 0.0596 \\
CSD-based & 0.1242 & 0.1288 & 0.1152 & 0.0733 & 0.0281 & 0.0512 \\
\hline
\end{tabular}



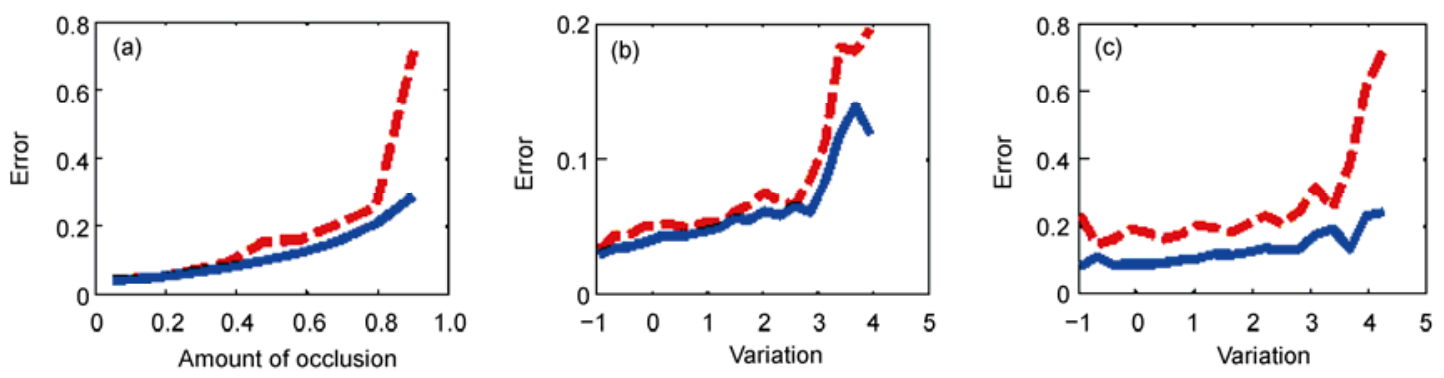

Figure 5 (a) The completion errors v.s. the amount of occlusion (in the log space); (b), (c) the completion errors v.s. the amount of shape variations (in the log space) of the observed parts under the same amount of occlusion $(R=0.25$ in (b) and $R=0.5$ in (c)). The red and blue curves are the results of GSD and CSD respectively.

shape variations result in the larger completion errors. Furthermore, we can also find from Figure 5 that, the errors climb slower for the CSD-based method than that of the GSD. This also demonstrates that, with the help of enough shape information and stronger prior model, the CSD-based method is more capable of accurately estimate the shape details under occlusion.

\subsection{Occluded-object detection by shape reconstruction}

We show how the proposed shape reconstruction methods facilitate object detection, contour localization and recovery in real occlusion images. First, we extract long salient curve segments in the edge map of a test image; these curves are considered as potential object contours, and matched to the reference shapes of our dictionary in section 1. Based on a voting scheme, we collect those curves which support for the same object (Figure 6(a)). Then we adopt the proposed shape completion methods to estimate the whole contours, given the supporting curves as observation. Finally, object hypotheses are verified according to the reconstruction probability and occlusion interpretations. Those largely recovered parts should be supported by occlusion boundaries in the image. Figure 6 (b),(c) show the detection results with recovered contours that originally occluded by other objects or missed due to image degradation. It is illustrated that the recovered shape are consistent with the observation and the object shape prior. Moreover, The CSD-based approach tends to produce more regular shapes because of the stronger shape prior constraints.

\subsection{Discussions}

With shape priors someone may suggest a simple idea by simply transforming a possible reference shape under only affine transformation for shape completion. This is a special case of our GSD-based method without estimating the optimal global wrapping deformation. However, it will result in the lost of shape variation details of different object individuals. Therefore, it is very necessary to infer the optimal shape variations in the proposed methods so that to achieve satisfying performances.

In the object detection applications, there is no need to compute the completed shapes under all the correspondences between the part and the whole object shape as in eq. (4). Due to the partial constraint as described above, only good partial matches are considered. We adopt e.g. the efficient partial shape matching approach in Riemenschneider et al.
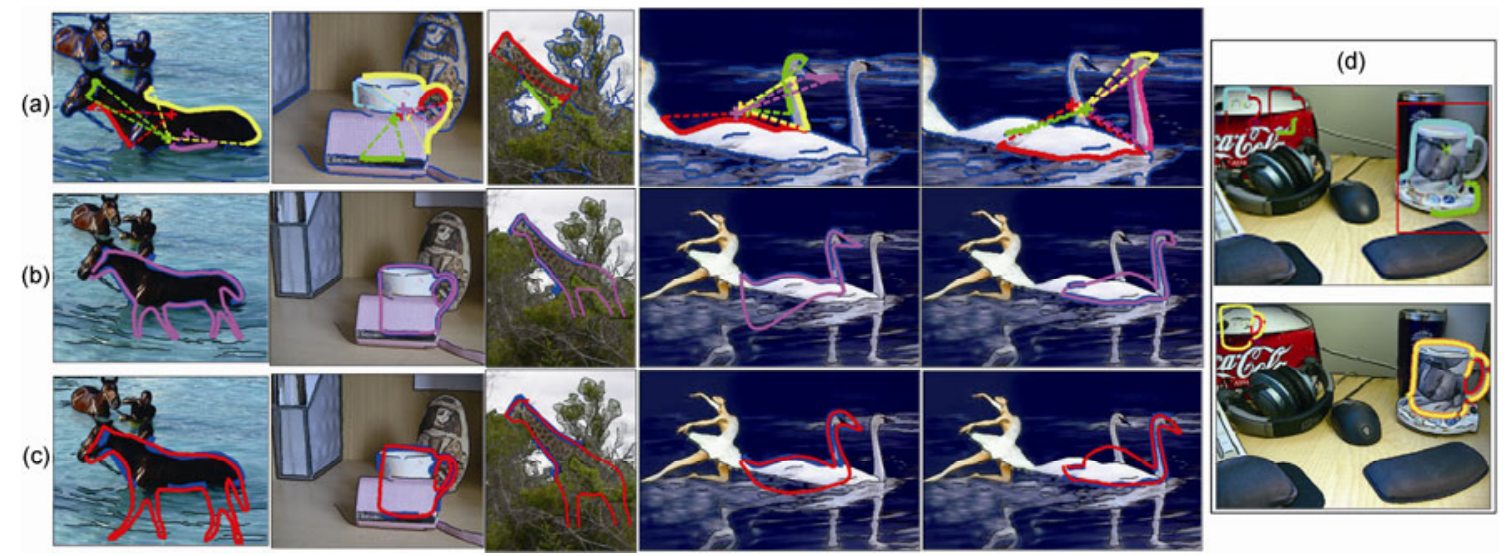

Figure 6 Object detection and contour completion. (a) The bold colorful solid lines show the observed collaborative curve segments to be completed; the ' $\mathrm{T}$ ' marks are the voted object centers and the blue thin curves are the edge maps; (b), (c) localized complete object contours given the observations as in (a), using the proposed GSD-based (in (b)) and CSD-based (in (c)) approaches respectively; (d) comparison of Riemenschneider et al. [28] (top) and our detection method (bottom). 
[28] to initially select possible good partial matches so as to reduce the computational cost. Although a voting-based detection method using independent curve segments is suggested in [28], there exist obvious disadvantages-as shown in Figure 6(d), our methods is able to detect more accurately localized complete contours, other than broken edge fragments and rough bounding boxes as in [28]. Moreover our contour completion provides good occlusion interpretations for object detection.

It should be noticed that the completion performance depends on the shape variation of the test instances (Figure 5). With the increase of the shape variation, the performance decreases. In a worst case the variation is out of the shape prior space, the result will greatly degrade. In addition, our methods are currently not very robust to the articulation of shape parts. Large articulation rather than shape deformation is another issue that should be considered in the future.

\section{Conclusions}

In this paper we suggest to draw on shape priors to reconstruct and complete object contours. The priors are established based on two kinds of shape databases, the GSD and CSD. The GSD provides prior knowledge of generic and common object shapes. The CSD provides class-specific shape priors by learning the shape models of each class. Accordingly, we propose two completion methods and compare their performances through the experiments. The completion accuracy of the CSD-based method is improved compared with the GSD-based method. Moreover, both the two methods achieve much better performances compared with the traditional curvature-based method such as the Euler spirals. In addition, it is found that the completion errors grow with the increase of the amount of occlusion and the shape variation of the observations.

Shape reconstruction is very useful for object detection and localization in real applications, especially in case of severe occlusion. Our experiments demonstrate the good performances of the occluded object detection based on the proposed shape completion methods.

In the future, we will research on the articulation models, the object poses and so on to further improve the shape completion and its applications in object detection, shape retrieval etc.

This work was supported by the National Basic Research Program of China (2009CB320904), the National Natural Science Foundation of China (61103087, 61121002 and 61272027) and the Scientific Research Foundation for the Returned Overseas Chinese Scholars, State Education Ministry.

1 Rensink R A, Enns J T. Early completion of occluded objects. Vision Res, 1998, 38: 489-2505
2 Rutkowski W S. Shape completion. Comput Vis Graph Image Process, 1979, 9: 89-101

3 Ullman S. Filling-in the gaps: The shape of subjective contours and a model for their generation. Vision Res, 1976, 25: 1-6

4 Brady M, Grimson W. Shape encoding and subjective contours. Annu Natl Conf Artif Intell. Stanford, CA. USA, 1980. 15-17

5 Horn B K P. The curve of least energy. ACM Trans Math Softw, 1983, 9: 441-460

6 Mumford D. Elastica and computer vision. Algebr Geom Its Appl, 1994, 491-506

7 Sharon E, Brandt A, Basri R. Completion energies and scale. IEEE Trans Pattern Anal and Mach Intell, 2000, 22: 1117-1131

8 Mio W, Srivastava A, Liu X. Contour inferences for image understanding. Int J Comput Vis, 2006, 33: 137-144

9 Kimia B, Frankel I, Popescu A. Euler spiral for shape completion. Int J Comput Vis, 2003, 54: 157-180

10 Ben-Yosef G, Ben-Shahar O. Minimum length in the tangent bundle as a model for curve completion. In: IEEE Int Conf Comput Vis and Pattern Recogn. San Francisco, CA. USA, 2010. 2384-2391

11 Venkatesh M V, Cheung S S. Symmetric shape completion under severe occlusions. IEEE Int Conf Image Process, 2006, Atlanta, GA, USA. 709-712

12 Kanizsa G, Gerbino W. Amodal completion: Seeing or thinking? J. Beck (Ed.), Organ Represent Percept, 1982, 167-190

13 Thornber K K, Williams L R. Characterizing the distribution of completion shapes with corners using a mixture of random processes. Pattern Recogn, 2000, 33: 543-553

14 Ren X F, Fowlkes C C, Malik J. Learning probabilistic models for contour completion in natural images. Int J Comput Vis, 2008, 77: 47-63

15 Wang S, Stahl J S, Bailey A, et al. Global detection of salient convex boundaries. Int J Comput Vis, 2007, 71: 337-359

16 Cremers D, Rousson M, Deriche R. A review of statistical approaches to level set segmentation: Integrating color, texture, motion and shape. Int J Comput Vis, 2007, 72: 195-215

17 Harary G, Tal A. 3D Euler spirals for 3D curve completion. In: ACM Symp Comput Geom. Snowbird. Utah, USA, 2010. 393-402

18 Sharf A, Alexa M, Cohen-Or D. Context-based surface completion. ACM Trans Graph, 2004, 23: 878-887

19 Ilic S, Fua P. Implicit meshes for modeling and reconstruction. In: IEEE Int Conf Comput Vis Pattern Recogn, Madison. WI, USA, 2003. 2: 483-490

20 Jenke $\mathrm{P}$, Wand $\mathrm{M}$, Bokeloh $\mathrm{M}$, et al. Bayesian point cloud reconstruction. Comput Graph Forum, 2006, 25: 337-359

21 Gal R, Shamir A, Hassner T, et al. Surface reconstruction using local shape priors. In: Eurographics Symp Geom Process. Barcelona, Spain, 2007. 253-262

22 Schnabel R, Degener P, Klein R. Completion and reconstruction with primitive shapes. Comput Graph Forum, 2009, 28: 503-512

23 Anguelov D, Srinivasan P, Koller D, et al. Scape: Shape completion and animation of people. ACM Trans Graph, 2005, 24: 408-416

24 Ferrari V, Tuytelaars T, Gool L V. Object detection by contour segment networks. Eur Conf Comput Vis, Graz, Austria, 2006, 14-28. Dataset: www.vision.ee.ethz.ch/ ferrari

25 Borenstein E, Sharon E, Ullman S. Combining top-down and bottom-up segmentation. IEEE Workshop Percept Organ Comput Vis, Washington, DC, USA, 2004, 46-53. Dataset: www.msri.org/people/ members/eranb/

26 Chui H, Rangarajan A. A new point matching algorithm for non-rigid registration. Comput Vis Image Understand, 2003, 89: 114-141

27 Cootes T F, Taylor C J, Cooper D H, et al. Active shape models their training and application. Comput Vis Image Understand, 1995, 61: 38-59

28 Riemenschneider H, Donoser M, Bischof H. Using partial edge contour matches for efficient object category localization. In: Eur Conf Comput Vis, Crete, Greece. 2010, Part V: 29-42

Open Access This article is distributed under the terms of the Creative Commons Attribution License which permits any use, distribution, and reproduction in any medium, provided the original author(s) and source are credited. 